\title{
Psychiatric and physical comorbidities and pain in patients with multiple sclerosis
}

This article was published in the following Dove Press journal: Journal of Pain Research

\author{
Rogier Scherder \\ Neeltje Kant ${ }^{2}$ \\ Evelien T Wolf ${ }^{3}$ \\ Bas Pijnenburg ${ }^{4}$ \\ Erik JA Scherder ${ }^{3}$ \\ 'Department of Orthopedics, \\ Westfries Gasthuis, Hoorn, \\ 2Department of Neuropsychology, \\ Reade, Amsterdam, ${ }^{3}$ Department \\ of Clinical Neuropsychology, Vrije \\ Universiteit, Amsterdam, ${ }^{4}$ Acibadem \\ International Medical Center. \\ Amsterdam, the Netherlands
}

Correspondence: EJA Scherder Department of Clinical Neuropsychology, Vrije Universiteit Amsterdam, Van der Boechorststraat I, 108I BT Amsterdam, the Netherlands

Tel +31 6I0391107

Email e.j.a.scherder@vu.nl_
Background: It has been observed that patients with multiple sclerosis (MS), who have psychiatric and physical comorbidities such as depression and COPD, have an increased risk of experiencing more pain. In this study, we have distinguished between pain intensity and pain affect, as the latter, particularly, requires treatment. Furthermore, while pain and comorbidities have been assessed using questionnaires, this is possibly a less reliable method for those who are cognitively vulnerable.

Objective: The aim of this study was to determine whether psychiatric and physical comorbidities can predict pain intensity and pain affect in MS patients, susceptible to cognitive impairment. Methods: Ninety-four patients with MS and 80 control participants participated in this crosssectional study. Besides depression and anxiety, 47 additional comorbidities were extracted from patients' medical records. Depression and anxiety were assessed using the Beck Depression Inventory and the Symptom Check List-90. Pain was assessed using the Number of Words Chosen Affective, Coloured Analog Scale, and the Faces Pain Scale. Cognitive functions, for example, memory and executive functions, were assessed using several neuropsychological tests. Results: The main findings indicate that psychiatric comorbidities (depression and anxiety) predict both pain intensity and pain affect and that total physical comorbidity predicts only pain affect in MS patients, susceptible to cognitive impairment.

Conclusion: Both psychiatric and physical comorbidities predict pain affect. All three clinical outcomes enhance MS patients' suffering.

Keywords: multiple sclerosis, pain, comorbidities, mood, cognition

\section{Introduction}

Although multiple sclerosis (MS) is categorized into four subtypes ( relapsing-remitting MS, primary-progressive MS, secondary-progressive MS, and progressive-relapsing MS), shared clinical symptoms include disturbances in motor activity (e.g., weakness and spasticity), sensory functioning (e.g., pain), visual functions (e.g., diplopia and optic neuritis), and cognition. ${ }^{1,2}$

However, clinical outcomes of MS show significant variation. ${ }^{3}$ One of the reasons for variations observed in clinical outcomes may be the comorbidity. ${ }^{3}$ It is well known that patients with MS suffer more from comorbid diseases compared to controls. ${ }^{4}$ More specifically, it has been shown that, irrespective of the subtype, the most prevalent comorbidities of MS include psychiatric comorbidities, such as depression and anxiety, and physical comorbidities, such as chronic lung disease, hypertension, hyperlipidemia, heart disease, and diabetes. ${ }^{3}$ Marrie $^{3}$ further states that the prevalence of "physical" 
comorbidities may increase further during the progression of the disease. In contrast, the prevalence of "psychiatric" comorbidities, such as depression, anxiety, schizophrenia, and bipolar disorder, may remain constant as has been observed in MS patients over a 10 -year period. ${ }^{5}$

It is clinically relevant to examine the influence of both psychiatric and physical comorbidities on the progression of MS, as some of the effects can be treated. ${ }^{6}$ For example, a close relationship between depression, anxiety, and pain has been observed in MS. ${ }^{7}$ In this Drulovic et $\mathrm{al}^{7}$ study, anxiety predicted pain. Depression may predict pain indirectly by predicting anxiety in MS patients. ${ }^{8}$ Pain in MS can be caused by painful conditions including osteoarthritis, lower back pain, migraines, spinal cord injury, and HIV infection ${ }^{9}$ or by the disease process itself, i.e., through demyelination of painrelated neuronal circuits. ${ }^{10}$ Concerning the central neuropathic pain, $\sim 30 \%$ of MS patients suffer from central/neuropathic pain, e.g., trigeminal neuralgia and migraines. ${ }^{11}$ Irrespective of its nature, pain has a negative influence on the quality of life and causes further physical disability. It is also associated with poorer sleep patterns and reduces the ability to work. ${ }^{12,13}$

In the present study, we have distinguished between pain intensity and pain affect. Patients seek medical help to reduce suffering, particularly in the case of pain affect. ${ }^{14}$ In the literature, pain intensity and pain affect have also been termed sensory-discriminative and motivational-affective aspects of pain, respectively. ${ }^{15}$ Within this scope, the sensorydiscriminative aspects of pain are related to pain threshold and motivational-affective aspects of pain to pain tolerance. ${ }^{15}$ In the central nervous system, the sensory-discriminative aspects are processed by the lateral pain system and motivational-affective aspects by the medial pain system. ${ }^{16}$ Based on the neuropathology, the medial pain system could be influenced in MS patients, and, as a result, these patients could experience more pain (pain affect). ${ }^{17}$ Because pain affect, particularly, is associated with chronic pain, we can argue that MS patients who suffer from chronic physical comorbidities also suffer more from pain affect.

Because of the assumed negative impact of physical comorbidities on pain affect, it is therefore potentially relevant to look not for possible psychiatric comorbidities such as depression and anxiety that may predict pain only. A number of studies have investigated the relationship between pain and comorbidity in MS. For example, MS patients with physical comorbidities, such as COPD as well as autoimmune and thyroid diseases, have an increased risk of experiencing more pain. ${ }^{18}$ In this latter study, osteoporosis and rheumatoid arthritis pain and comorbidities were assessed using a validated questionnaire. ${ }^{18}$ The "Self-Report Comorbidity Questionnaire"19 refers to medical terms such as Sjögren's syndrome and thyroid disease, and the results show that the patients were capable of answering this questionnaire. There was no indication that the patients were cognitively impaired, which is important when answering a questionnaire. However, for MS patients, susceptible to cognitive impairment, this method of gathering information might be more difficult as cognitive decline is present in $\sim 54 \%-65 \%$ of MS patients. ${ }^{20}$

Therefore, in the present study with MS patients, susceptible to cognitive impairment, we carefully monitored medical records for the presence of comorbidities. In Fiest et al's study, ${ }^{18}$ pain was assessed using another questionnaire - the Health Utilities Index (Mark III, version). We administered Visual Analog Scales, Faces Pain Scale, and a list of words that characterize the nature of the pain experienced by the patient. Although the validity of these scales has not yet been examined in patients with MS, combination of these scales has been used in various studies, including cognitively impaired patients. For example, in one study, $75 \%-100 \%$ of patients in the early stages of Alzheimer's disease who participated were able to comprehend the concept of the scales. ${ }^{21}$ The results observed in our studies when administering Visual Analog Scales and Faces Pain Scale to Alzheimer patients suggest an unchanged pain threshold and an increased pain tolerance, a distinction that was confirmed in a study applying experimental pain stimuli, i.e., electrical stimulation and ischemia. ${ }^{22}$ Finally, a Visual Analog Scale was used in a previous study on pain in MS patients. ${ }^{7}$

To summarize, the goal of the present study was to investigate the relationship between pain intensity, pain affect, and comorbidities in MS patients, susceptible to cognitive impairment, by assessing pain intensity and pain affect by Visual Analog Scales and a Verbal Pain Scale, and by mapping the comorbidities through medical records. A positive relationship between pain - and pain affect in particular - and comorbidities is expected.

\section{Methods \\ Study design}

This cross-sectional study is part of a larger study investigating the relationship between pain and cognition in MS.

\section{Participants}

The current study consisted of 94 patients with MS and 80 control participants, matched for age. The control group served as a reference group concerning cognitive functioning, comorbidities, pain, and mood. A neurologist diagnosed all patients. MS patients were enrolled and examined either at 
Nieuw Unicum, a center for the professional community care of clients with primarily physical disabilities, Zandvoort, the Netherlands, or from the personal environment of the investigators. The control participants were also enrolled from the personal environment of the investigators. We performed a power analysis, with a set $\alpha$ of 0.05 and $\beta$ of 0.80 , and expected small to moderate effect sizes of regression coefficients for the main predictors (mood and comorbidities) on the main outcome parameters (pain intensity and pain affect), resulting in a required minimal sample size of 72 . In each group, we included more patients to accommodate possible dropouts.

The principal investigator trained each student extensively, to maximize interrater reliability in administration of the tests. For more details regarding the age of participants and for data on the percentages of male and female participants, see Table 1.

\section{Education}

Both the MS patients and the control participants were screened for education. Education was divided into five categories: elementary school not finished (score $=1$ ), elementary school (score=2), lower secondary school (score=3), higher secondary school (score $=4$ ), and higher vocational training for $18+$ years/university (score $=5$ ). For data analysis, see Table 1.

\section{Global cognitive functioning}

Global cognitive functioning was assessed using the Mini Mental State Examination (MMSE). ${ }^{23}$ Scores are presented as percentage. This procedure is meant to obtain a reliable MMSE score, as the number of MMSE items that had to be left out differed for each patient due to motor disturbances (minimum score $=0$, maximum score $=100$ ). A percentage $<83 \%$ indicates cognitive decline (a score of $83 \%$ is identical to a standard MMSE score of 25, with a maximum score of 30). There was no minimum threshold of available items. For data analysis, see Table 1.

\section{Specific cognitive functions}

For verbal long-term memory, we administered the EightWords Test. ${ }^{24}$ This test comprises three conditions: immediate recall, delayed recall, and recognition. For nonverbal longterm memory, the Rivermead Behavioural Memory Test Faces and Pictures was applied. ${ }^{25}$ Executive functions were also assessed: set-shifting using Word Fluency and Rule-Shift Cards, working memory using Digit Span Backward, and planning using Key Search Test. These tests are part of the Behavioural Assessment of the Dysexecutive Syndrome. ${ }^{26}$ For further details, see the study by Scherder et al. ${ }^{27}$ Data are presented in Table 1.

\section{Exclusion criteria}

Participants were excluded if they had a history of alcoholism, cerebral trauma, normal pressure hydrocephalus, disorders of the central nervous system apart from MS, neoplasms, or disturbances of consciousness.

Table I Age, education, the MMSE, assessing global cognitive functioning and neuropsychological tests that assess memory and executive functions

\begin{tabular}{|c|c|c|c|c|c|c|c|c|c|}
\hline \multirow{2}{*}{$\begin{array}{l}\text { Demographics, global and specific } \\
\text { cognitive functions, analgesics }\end{array}$} & \multicolumn{3}{|c|}{ MS group } & \multicolumn{3}{|c|}{ Control group } & \multicolumn{3}{|c|}{ Statistics } \\
\hline & $\mathbf{N}$ & $\%$ & SD & $\overline{\mathbf{N}}$ & $\%$ & SD & $\overline{\chi^{2}}$ & & $p$-value \\
\hline \multirow[t]{2}{*}{ Male } & $35 / 94$ & 37.2 & & $27 / 80$ & 33.8 & & 0.23 & & $<0.64$ \\
\hline & $\mathbf{N}$ & $\mathbf{M}$ & SD & $\mathbf{N}$ & $\mathbf{M}$ & SD & $\mathbf{T}$ & $d f$ & $p$-value \\
\hline Age & 94 & 51.06 & 9.51 & 80 & 48.79 & 10.49 & 1.50 & 172 & $<0.14$ \\
\hline Education & 89 & 3.51 & 0.76 & 80 & 3.50 & 0.78 & 0.05 & 167 & $<0.97$ \\
\hline MMSE (\%) & 94 & 89.64 & 12.37 & 80 & 97.40 & 3.84 & 5.39 & 172 & $<0.001$ \\
\hline \multicolumn{10}{|l|}{ Memory } \\
\hline \multicolumn{10}{|l|}{ Eight-Words Test } \\
\hline Immediate recall & 93 & 29.32 & 6.78 & 78 & 34.08 & 3.83 & 5.50 & 169 & $<0.001$ \\
\hline Delayed recall & 94 & 4.38 & 2.44 & 78 & 6.46 & 1.42 & 6.64 & 170 & $<0.001$ \\
\hline Recognition & 94 & 14.59 & 2.30 & 78 & 15.67 & 0.85 & 3.94 & 170 & $<0.001$ \\
\hline RBMT faces & 88 & 13.68 & 5.89 & 80 & 18.23 & 2.62 & 6.35 & 166 & $<0.002$ \\
\hline RBMT pictures & 91 & 36.00 & 6.70 & 80 & 38.60 & 2.14 & 3.32 & 169 & $<0.001$ \\
\hline \multicolumn{10}{|l|}{ Executive functions } \\
\hline Word Fluency & 89 & 35.28 & 14.03 & 80 & 44.10 & 11.04 & 4.51 & 167 & $<0.001$ \\
\hline Rule-Shift Cards & 88 & 3.03 & 1.22 & 80 & 3.46 & 0.86 & 2.61 & 166 & $<0.02$ \\
\hline Digit Span Backward & 92 & 5.13 & 2.07 & 71 & 6.24 & 1.90 & 3.52 & 161 & $<0.002$ \\
\hline Key Search Test & 79 & $1 \mathrm{l} .42$ & 3.62 & 80 & 11.50 & 3.46 & 0.15 & 157 & $<0.89$ \\
\hline
\end{tabular}

Abbreviations: $d f$, degrees of freedom; M, mean; MS, multiple sclerosis; MMSE, Mini Mental State Examination; RBMT, Rivermead Behavioural Memory Test. 


\section{Medication}

The use of the following analgesics was noted (score $0=$ no use, score $1=$ in use) to evaluate the possible influence on pain experience: baclofen, paracetamol, diclofenac, naproxen, ibuprofen, and cannabis. Besides pain medication, medication prescribed for major comorbidities, such as cardiovascular diseases, respiratory diseases, and gastrointestinal diseases, was also noted. Only pain medication was included in data analysis (Table 2).

\section{Informed consent}

The study was approved by the VU Medical Centre's Medical Ethical Committee. The MS patients and control participants were extensively informed about the aims and procedures of the investigation, and they were asked to sign an informed consent form. After obtaining permission, neuropsychological tests, vital sensibility tests, and pain perception tests were performed.

In addition, the study has been carried out in accordance with the Code of Ethics of the World Medical Association (Declaration of Helsinki).

\section{Materials and procedure Mood}

Depression and anxiety are factors that are already known to be related to pain experience. ${ }^{28}$ Therefore, in the present study, mood was analyzed separately from the other comorbidities. Three questionnaires were administered: the
Beck Depression Inventory (minimum score $=0$, maximum score $=63$ ), ${ }^{29,30}$ Symptom Check List-90 (SCL-90) anxiety subscale (minimum score $=0$, maximum score $=40),{ }^{31}$ and SCL-90 depression subscale (minimum score $=0$, maximum score $=52) .{ }^{32}$ The scores of these three scales were converted into z-scores to obtain a composite domain score for mood (Cronbach's $\alpha=0.85$ ). See Table 3 for data analysis.

\section{Pain}

Number of Words Chosen-Affective (NWC-A)

The NWC-A ${ }^{33}$ is part of the McGill Pain Questionnaire (Dutch version) and it addresses the affective components of pain. This subscale is composed of five separate subgroups of three affective words. The participants are asked to select one word out of each subgroup that best describes his or her pain (minimum score $=0$, maximum score $=15$ ). As our patients might suffer from a mild to moderate decline in both cognition and motor capacities, we did not use other parts of the McGill Questionnaire, as they appeal either to memory ("pain on most days") or fine motor functions (Visual Analog Scale).

\section{Coloured Analog Scale (CAS)}

The $\mathrm{CAS}^{34}$ is a Visual Analog Scale applied in two forms: one measures the intensity of pain (CAS intensity) and the other measures the suffering due to pain (CAS affect). It has a plastic slide that can be moved from the bottom (no pain: light pink color) to the top (worst pain: dark red color) to assess the participant's pain. The scores are converted to

Table 2 Percentages and chi-square tests regarding pain medication in both MS group and controls

\begin{tabular}{|c|c|c|c|c|c|c|}
\hline \multirow[t]{2}{*}{ Pain medication } & \multicolumn{2}{|c|}{ MS group } & \multicolumn{2}{|c|}{ Controls } & \multicolumn{2}{|c|}{ Statistics } \\
\hline & $\mathbf{N}$ & $\%$ & $\mathbf{N}$ & $\%$ & $\chi^{2}$ & Fisher's exact test \\
\hline Baclofen & 25 & 33.8 & 0 & 0 & 27.88 & 0.001 \\
\hline Paracetamol & 20 & 27.0 & $\mathrm{I}$ & $\mathrm{I} .5$ & 18.37 & 0.001 \\
\hline Diclofenac & 3 & 4.0 & 2 & 2.9 & 0.12 & 1.00 \\
\hline Naproxyn & I & 1.4 & 0 & 0 & 0.93 & 1.00 \\
\hline Ibuprofen & 3 & 4.1 & 0 & 0 & 2.82 & 0.25 \\
\hline Cannabis & 4 & 13.3 & 0 & 0 & 10.53 & 0.007 \\
\hline
\end{tabular}

Abbreviation: MS, multiple sclerosis.

Table 3 Means, SDs, and Mann-Whitney $U$ tests regarding depression and anxiety in patients with multiple sclerosis (MS) and persons without MS (controls)

\begin{tabular}{|c|c|c|c|c|c|c|c|c|}
\hline \multirow[t]{2}{*}{ Mood } & \multicolumn{3}{|c|}{ MS group } & \multicolumn{3}{|c|}{ Controls } & \multicolumn{2}{|c|}{ Mann-Whitney $U$ test } \\
\hline & $\mathbf{N}$ & $\mathbf{M}$ & SD & $\mathbf{N}$ & $\mathbf{M}$ & SD & $\mathbf{Z}$ & $p$-value \\
\hline BDI & 92 & 7.58 & 5.07 & 79 & 4.75 & 4.39 & -4.28 & $<0.001$ \\
\hline SCL-90 anxiety & 92 & 13.86 & 4.58 & 80 & 11.89 & 2.47 & -3.25 & $<0.001$ \\
\hline SCL-90 depression & 93 & 22.74 & 7.47 & 80 & 18.74 & 4.47 & -4.32 & $<0.001$ \\
\hline Mood domain & 91 & 44.33 & 15.17 & 79 & 35.38 & 9.82 & -4.59 & $<0.001$ \\
\hline
\end{tabular}

Abbreviations: BDI, Beck Depression Inventory; M, mean; SCL-90, Symptom Check List-90. 
a $0-10$ scale $(0=$ no pain, $10=$ severe pain) to measure the intensity of pain or suffering due to pain.

\section{Faces Pain Scale (FPS)}

The MS patients and control participants indicated how much pain they experienced by choosing one of seven different faces, which all expressed different levels of pain $^{35}(0=$ no pain, $6=$ severe pain). A participant was considered to be "pain free" when the score was zero on all four pain scales.

The scores of these four scales were converted into Z-scores to obtain a composite domain score for pain (Cronbach's $\alpha=0.93$ ). To examine a possible relationship between pain and comorbidity in more detail, two pain subdomains were composed within the group of MS patients experiencing pain. The scores of the CAS intensity and FPS were converted into z-scores to obtain a pain domain "intensity" (Cronbach's $\alpha=0.89$ ), whereas the scores of the CAS suffering and NWC-A were converted into z-scores to obtain a pain domain "affect" (Cronbach's $\alpha=0.83$ ). For data analysis, see Table 4.

\section{Comorbidity}

The following comorbidities were taken into account: cardiovascular diseases (arteriosclerosis, arrhythmias,

Table 4 Adjusted means and SDs regarding pain affect and pain intensity in patients with MS and in persons without MS

\begin{tabular}{|c|c|c|c|c|c|c|}
\hline \multirow[t]{2}{*}{ Pain affect } & \multicolumn{3}{|c|}{ MS group } & \multicolumn{3}{|c|}{ Controls } \\
\hline & $\mathbf{N}$ & $M$ & SD & $\mathbf{N}$ & $M$ & SD \\
\hline CAS affect & 90 & 2.63 & 2.93 & 77 & 0.86 & 1.84 \\
\hline NWC-A & 91 & 3.85 & 4.07 & 79 & 1.09 & 2.02 \\
\hline Domain & 87 & 6.48 & 6.36 & 76 & 1.96 & 3.44 \\
\hline \multicolumn{7}{|l|}{ Pain intensity } \\
\hline CAS intensity & 93 & 2.94 & 2.79 & 79 & 1.02 & 1.83 \\
\hline FPS & 84 & 1.90 & 1.81 & 79 & 0.63 & 1.16 \\
\hline Domain & 74 & 4.88 & 4.31 & 79 & 1.65 & 2.81 \\
\hline
\end{tabular}

Abbreviations: CAS, Coloured Analog Scale; FPS, Faces Pain Scale; M, mean; MS, multiple sclerosis; NWC-A, Number of Words Chosen Affective. hypertension, hypotension, deep vein thrombosis, peripheral vascular diseases, congestive heart failure), endocrinological and metabolic disorders (hyperthyroidism, hypothyroidism, diabetes mellitus), neurological disorders (aphasia, transient ischemic attack, cerebrovascular accident, hemiplegia, quadriplegia, paraplegia, epilepsy, traumatic brain injury, migraine), eye disorders (cataract, diabetic retinopathy, glaucoma, macular degeneration), infections (antibiotic resistant infection, clostridium difficile colitis, HIV infection, pneumonia, airway infection, sepsis, sexually transmitted diseases, conjunctivitis, urinary tract infection, viral hepatitis, wound infection), internal disorders (asthma, COPD/emphysema, renal failure, anemia, gastrointestinal disorders), disorders of the musculoskeletal system (movement disorders, rheumatic disorders, hip fracture, osteoporosis, pathological bone fracture, limb amputation), and psychiatric disorders other than mood (schizophrenia, bipolar disorder). Each separate comorbidity was scored as follows: $0=$ absent, $1=$ present. All scores were added up, providing a total comorbidity score. In Table 5, results of the data analysis are presented.

\section{Procedure}

The mood and pain scales were administered at the same time in a single session. The total duration of the administration of the questionnaires and scales was $\sim 30 \mathrm{~min}$. All participants were able to complete the scales and questionnaires. In terms of pain, the subjects were asked about their level of pain during the last week.

\section{Data analyses}

The SPSS-PC program was used for data analyses. First, possible differences between both groups concerning pain and mood were analyzed by means of a multivariate analysis of covariance (MANCOVA), with mood as a covariate. Possible differences between both groups concerning pain medication and comorbidity were analyzed by means of chi-square tests. As some cells had a low cell count, especially in the control

Table 5 Percentages and chi-square tests regarding comorbidity in patients with multiple sclerosis (MS) and persons without MS (controls)

\begin{tabular}{|c|c|c|c|c|c|c|}
\hline \multirow[t]{2}{*}{ Physical comorbidities } & \multicolumn{2}{|c|}{ MS group } & \multicolumn{2}{|c|}{ Controls } & \multicolumn{2}{|c|}{ Statistics } \\
\hline & $\mathbf{N}$ & $\%$ & $\mathbf{n}$ & $\%$ & $\chi^{2}(1)$ & Fisher's Exact Test \\
\hline Cardiovascular diseases & 8 & 10.8 & 8 & 12.3 & 0.08 & 0.80 \\
\hline Endocrinological/metabolic disorders & 6 & 8.1 & 2 & 3.1 & 1.62 & 0.29 \\
\hline Neurological disorders & 5 & 6.8 & 3 & 4.6 & 0.29 & 0.73 \\
\hline Eye disorders & 0 & 0.0 & 1 & 1.5 & 1.15 & 0.47 \\
\hline Infections & 5 & 6.8 & 2 & 3.1 & 0.98 & 0.45 \\
\hline Internal disorders & 6 & 8.1 & 9 & 13.8 & 1.18 & 0.30 \\
\hline Musculoskeletal disorders & 6 & 8.2 & 19 & 29.2 & 10.23 & 0.003 \\
\hline
\end{tabular}


group, statistical significance was established by means of Fischer's exact tests (two-tailed). Mann-Whitney $U$ tests were applied for mood and Student's $t$-tests for analyzing differences in age and scores on specific cognitive tests. The relationship between pain and comorbidity was analyzed by a hierarchical linear regression analysis, one model predicting pain intensity and one predicting pain affect. In the first step, mood was added as a predictor, and in the second step, the total comorbidity domain was entered. Adjusted $R^{2}$ and $R^{2}$ are reported as measures of model fit and $\Delta R^{2}$ as a measure of the effect of comorbidity on pain while controlling for mood. A significance level of $p<0.05$ was applied.

\section{Results}

\section{Demographics, global and specific cognitive functioning, and use of \\ analgesics}

As can be seen in Table 1, the groups did not differ significantly in terms of age, gender, and education. However, both groups showed a significant difference regarding global cognitive functioning, measured by the MMSE. In addition, MS patients scored significantly lower on all tests for memory and executive functions, except on the Key Search Test.

\section{Pain medication}

Data analyses using chi-square tests showed that the MS patients used significantly more baclofen, paracetamol, and cannabis compared to the controls (see Table 2 for percentages, chi-square, and Fisher exact tests).

\section{Psychiatric comorbidities: mood domain}

Psychiatric comorbidities other than mood, i.e., schizophrenia and bipolar disorders, were not present in both groups. Therefore, we analyzed depression and anxiety. Mann-Whitney $U$ tests revealed that the patients with MS suffered significantly more from depression and anxiety than the control group (for means, SDs, and Mann-Whitney $U$ tests for separate scales and domains, see Table 3).

\section{Pain experience}

A MANCOVA, with mood domain as a covariate, showed a main effect for group $(F[2,146]=7.36, p<0.002)$. Univariate $F$-tests showed similar findings, with pain intensity domain $(F[1,138]=18.73, p<0.001)$ and pain affect domain $(F[1,138]=15.99, p<0.001)$. Adjusted mean scores (Table 4) showed that MS patients experienced significantly more pain affect and pain intensity when controlled for mood than the controls.

\section{Total and separate physical comorbidities}

The total physical comorbidity domain differed significantly between both groups (chi-square $=24.09, d f=7, p<0.002$ ). As can be seen from Table 5, illustrating each separate physical comorbidity, only musculoskeletal disorders (movement disorders other than MS, rheumatic disorders, hip fracture, osteoporosis, pathological bone fracture, limb amputation) occurred significantly more in the controls $(p<0.005)$.

\section{Relationship between total comorbidity, mood, and pain in MS patients}

\section{Pain intensity domain}

In Model 1, mood was the significant predictor of pain intensity $(p<0.02)$, whose variance was $11 \%$. Adding total comorbidity as a predictor in Model 2 did not significantly increase the explained variance (Table 6).

\section{Pain affect domain}

In Model 1, similar to the pain intensity domain, data analyses showed that mood significantly explained $10 \%$ of the variance $(p<0.02)$ (Table 7), and in Model 2, comorbidity was added as a predictor. Comorbidity significantly explained $6 \%$ of the variance in pain affect (Table 7).

\section{Discussion}

The main goal of the present study was to examine whether a positive relationship exists between psychiatric and physical comorbidities, pain intensity, and pain affect in MS patients.

Table 6 Hierarchical regression analysis predicting pain intensity (dependent variable) with mood and total physical comorbidity as predictors in a subsample of patients with multiple sclerosis $(\mathrm{N}=62)$

\begin{tabular}{|c|c|c|c|c|c|c|c|c|c|}
\hline Pain intensity & $\beta(\mathrm{SE})$ & $\mathbf{t}$ & $p$-value & $F$ & $d f$ & $p$-value & $R_{\text {adj }}^{2}$ & $R^{2}$ & $\Delta R^{2}$ \\
\hline Model I & & & & 5.02 & 1.60 & 0.03 & 0.06 & 0.08 & $\mathrm{n} / \mathrm{a}$ \\
\hline Mood & $0.28(0.09)$ & 2.24 & 0.03 & & & & & & \\
\hline Model 2 & & & & 1.03 & 1.59 & 0.32 & 0.06 & 0.09 & 0.02 \\
\hline Mood & $0.26(0.09)$ & 2.10 & 0.04 & & & & & & \\
\hline Total physical comorbidity & $0.13(0.16)$ & 1.01 & 0.32 & & & & & & \\
\hline
\end{tabular}

Abbreviations: $d f$, degrees of freedom; $n / a$, not applicable; SE, standard error. 
Table 7 Hierarchical regression analysis predicting pain affect (dependent variable) with mood and total physical comorbidity in a subsample of patients with $M S(N=67)$

\begin{tabular}{|c|c|c|c|c|c|c|c|c|c|}
\hline Pain affect & $\beta(\mathrm{SE})$ & $\mathbf{t}$ & $p$-value & $\boldsymbol{F}$ & $d f$ & $p$-value & $R_{\text {adj }}^{2}$ & $R^{2}$ & $\Delta R^{2}$ \\
\hline Model I & & & & 4.21 & 1.65 & 0.04 & 0.05 & 0.06 & $\mathrm{n} / \mathrm{a}$ \\
\hline Mood & $0.25(0.08)$ & 2.05 & 0.04 & & & & & & \\
\hline Model 2 & & & & 5.76 & 2.62 & 0.005 & 0.13 & 0.16 & 0.06 \\
\hline Mood & $0.24(0.08)$ & 2.02 & 0.05 & & & & & & \\
\hline Total physical comorbidity & $0.27(0.14)$ & 2.35 & 0.02 & & & & & & \\
\hline
\end{tabular}

Abbreviations: $d f$, degrees of freedom; MS, multiple sclerosis; $n / a$, not applicable; SE, standard error.

If so, the mere presence of comorbidities could alert medical staff to the presence of pain, especially in those MS patients who are cognitively vulnerable.

As psychiatric comorbidities such as schizophrenia and bipolar disorders were not present in the MS group, we analyzed whether psychiatric comorbidity mood, consisting of anxiety and depression, and total physical comorbidity could predict pain intensity, pain affect, or both.

The main findings of this study are twofold. First, mood appears to be a significant predictor of both pain intensity and pain affect. A close relationship between mood and pain in MS has been observed in an earlier study. ${ }^{7}$ In their study, Drulovic et $\mathrm{al}^{7}$ used a numerical Visual Analog Scale to assess the intensity of pain (there was no separate assessment of pain affect). A close relationship between pain and depression, for example, is also reflected in the analgesic effect of antidepressive medication, ${ }^{36}$ and in the anti-depressive effect of analgesics, e.g., Tramadol, in patients with lower back pain. ${ }^{37}$ In MS, anti-depressants are administered for pain treatment in general. ${ }^{38}$ Apart from its relationship with pain, depression and anxiety should be the primary focus of treatment from the onset of diagnosis, considering the high incidence. ${ }^{3}$

The second finding shows that total physical comorbidity was only a significant predictor of pain affect and not of pain intensity. A possible underlying mechanism for this could be that both physical comorbidities, such as hypertension and chronic lung disease, and chronic pain are considered stressful events, which consequently may increase the burden of MS patients. ${ }^{39,40}$ Suffering from pain (i.e., pain affect) is known to be stressful, particularly when it is not adequately controlled. ${ }^{41}$ In the Janssen et al study, not being able to manage pain caused several stress-related physiological reactions, like an increase in heart rate response. However, it did not increase pain intensity. With regard to pain, the present findings show that MS patients experience significantly more pain intensity and pain affect than persons without MS, despite the fact that they used significantly more analgesics. The majority of the MS patients used baclofen (33.8\%), which is understandable considering the high prevalence of central/neuropathic pain and tonic spasms in MS. ${ }^{11}$ The same holds for cannabis. ${ }^{42}$

More remarkable is that $27 \%$ of the MS patients used paracetamol (acetaminophen), most commonly prescribed for joint pain and myalgia, ${ }^{43}$ which is not effective in the treatment of central/neuropathic pain. It is unlikely that the patients in our sample suffered mostly from joint pain and myalgia, as musculoskeletal disorders (mainly neck and shoulder pain, lower back pain, muscle aches) occurred significantly more frequently in the control group. This might partly explain why the MS patients in the present study still indicated that they suffered significantly more from pain than those without MS. More adequate pain treatment preventing the possible undertreatment of pain can be achieved by increasing patients' self-efficacy and own pain management, among other methods. ${ }^{44}$

Although the distinction made in this study between pain intensity and pain affect seems somewhat arbitrary, the distinction is a known phenomenon in pain research. In the brain, areas involved in the processing of pain intensity, e.g., primary and secondary somatosensory cortices (lateral pain system), are distinct from those processing the affective components of pain, e.g., the parahippocampal gyrus and the amygdala (medial pain system). ${ }^{45}$ Experimental pain studies also show a distinction between both aspects of pain. For example, heat was applied to healthy adults to assess pain intensity and pain affect. ${ }^{46}$ The results suggest that those suffering less from pain affect scored higher on a resilience scale. Interestingly, in a study conducted by Carvalho et $\mathrm{al}^{47}$ it was found that depression and anxiety can lower patients' resilience, especially in patients suffering from cardiovascular disease. Within the scope of our study of MS patients, we, therefore, argue that effective treatment of depression and anxiety may enhance resilience, with a positive effect on pain affect.

Finally, we included MS patients who were cognitively vulnerable. For cognitively "unimpaired" MS patients, application of a questionnaire such as the "Self-Report 
Comorbidity Questionnaire"19 appeared to be most adequate. ${ }^{18}$ However, such a questionnaire appeals to, among other aspects, long-term episodic memory. For example, the patient must remember the year of the diagnosis and the exact names of the diagnosis, e.g., Sjögren's syndrome, anemia, and degenerative arthritis. These names might have been explicitly mentioned to them a number of years ago. The participants in our study showed a significant impairment in episodic memory compared to the control group (Table 1). For that reason, we carefully monitored the medical records for the presence of both psychiatric and physical comorbidities. We applied Visual Analog Scales and questionnaires in the present study to assess pain intensity and affect as well as depression and anxiety. However, these data collection instruments are not suitable for long-term memory, but they assess pain experienced during the last week only. Moreover, these instruments have been applied to $\mathrm{MS}$ patients ${ }^{7}$ and patients in the early stages of dementia. ${ }^{21}$ The reliability of these instruments has been confirmed in an experimental pain study. ${ }^{22}$

\section{Limitations}

The first limitation of the present study is that we did not differentiate between the various subtypes of MS nor could we provide the Expanded Disability Status scale (EDSS) ${ }^{48}$ or disease duration for all patients. Unfortunately, this information was not always available in the medical records. An attenuating circumstance is that data from a comprehensive meta-analysis ${ }^{49}$ suggest that the various subtypes of MS do not differ with respect to the prevalence of overall pain and headaches. However, the authors emphasize that the number of studies included was limited and more studies are needed. Indeed, one could expect a difference in pain experience between the various subgroups of MS. The cognitive profiles differ between chronic-progressive and relapsing-remitting subtypes, ${ }^{50}$ for example, and the neuronal systems that are involved in cognitive processes show considerable overlap with the neuronal systems processing pain. ${ }^{17}$ Concerning the relationship between EDSS, disease progression, and pain, Foley et $\mathrm{al}^{49}$ described a study in which the prevalence of pain increased over a period of 5 years, particularly in patients with a declining EDSS scale. ${ }^{51}$ In that study, no distinction between various subgroups of MS was made.

The second limitation might be the use of Visual Ana$\log$ Scales, Faces Pain Scale, and Verbal Pain Affect Scale, although they have been used in previous studies involving cognitively impaired patients and also in a previous study on pain in MS. ${ }^{7}$ For future studies, we recommend that more reliable methods, such as Quantitative Sensory Testing and heat and cold threshold determination, be used.
The third limitation might be that non-MS instruments were used to assess cognition. In particular, the MMSE appears to be less sensitive for cognitive impairment in MS than other global cognitive assessment tools, ${ }^{52}$ such as the Brief International Cognitive Assessment for Multiple Sclerosis (BICAMS). ${ }^{53}$ The BICAMS consists of three neuropsychological tests: Symbol Digit Modalities Test (which only takes $5 \mathrm{~min}$ to complete) and, if more time is available, the California Verbal Learning Test and the Brief Visuospatial Memory Test (which takes an additional $10 \mathrm{~min}$ to complete). In the present study, in the context of a larger study on the relation between pain and cognition in MS, we selected a more extensive neuropsychological test battery to answer specific research questions.

The fourth limitation is that we did not control for fatigue. Not only does fatigue occur in the majority of MS patients - it is considered to be one of the most disabling symptoms of $\mathrm{MS}^{54}$ - but a relationship between fatigue, pain, and cognition has also been previously described. ${ }^{55}$ A positive relationship between pain and fatigue, and a negative relationship between cognitive functioning and both pain and fatigue, was observed. ${ }^{55}$ Within the scope of the present study, it would be interesting to examine whether the relationship between fatigue and pain affect is stronger than between fatigue and pain intensity in MS patients.

Finally, it would have been more informative if we had identified various types of pain, according to the classification proposed by O'Connor et al, ${ }^{56}$ such as trigeminal neuralgias, migraine, painful tonic spasm, back pain, and headache. The clinical relevance of such a classification is that each type of pain might require a different treatment approach.

\section{Conclusion}

Psychiatric and physical comorbidities and pain affect may enhance MS patients' suffering. Assessment of the various aspects of pain is complex, particularly in MS patients, susceptible to cognitive impairment. Our findings might therefore be of relevance for the clinician, to optimize pain assessment and treatment.

\section{Disclosure}

The authors report no conflicts of interest in this work.

\section{References}

1. Katz Sand I. Classification, diagnosis, and differential diagnosis of multiple sclerosis. Curr Opin Neurol. 2015;28(3):193-205.

2. Milo R, Miller A. Revised diagnostic criteria of multiple sclerosis. Autoimmun Rev. 2014;13(4-5):518-524.

3. Marrie RA. Comorbidity in multiple sclerosis: implications for patient care. Nat Rev Neurol. 2017;13(6):375-382. 
4. Capkun G, Dahlke F, Lahoz R, et al. Mortality and comorbidities in patients with multiple sclerosis compared with a population without multiple sclerosis: an observational study using the US Department of Defense administrative claims database. Mult Scler Relat Disord. 2015;4(6):546-554.

5. Marrie RA, Reider N, Cohen J, et al. A systematic review of the incidence and prevalence of cardiac, cerebrovascular, and peripheral vascular disease in multiple sclerosis. Mult Scler. 2015;21(3):318-331.

6. Amato MP, Derfuss T, Hemmer B, et al; 2016 ECTRIMS Focused Workshop Group. Environmental modifiable risk factors for multiple sclerosis: report from the 2016 ECTRIMS focused workshop. Mult Scler. 2017;1352458516686847.

7. Drulovic J, Basic-Kes V, Grgic S, et al. The prevalence of pain in adults with multiple sclerosis: a multicenter cross-sectional survey. Pain Med. 2015;16(8):1597-602.

8. Hartoonian N, Terrill AL, Beier ML, et al. Predictors of anxiety in multiple sclerosis. Rehabil Psychol. 2015;60(1):91-98.

9. Davis JA, Robinson RL, Le TK, Xie J. Incidence and impact of pain conditions and comorbid illnesses. J Pain Res. 2011;4:331-345.

10. Solaro C, Trabucco E, Messmer Uccelli M. Pain and multiple sclerosis: pathophysiology and treatment. Curr Neurol Neurosci Rep. 2013; 13(1):320

11. Mazhari A. Multiple-sclerosis related pain syndromes: an imaging update. Curr Pain Headache Rep. 2016;20(12):63.

12. Solaro C, Uccelli MM. Management of pain in multiple sclerosis: a pharmacological approach. Nat Rev Neurol. 2011;7(9):519-527.

13. Truini A, Galeotti F, Cruccu G. Treating pain in multiple sclerosis. Expert Opin Pharmac. 2011;12(15):2355-2368.

14. Sewards TV, Sewards MA. The medial pain system: neural representations of the motivational aspect of pain. Brain Res Bull. 2002;59(3): $163-180$.

15. Scherder EJ, Sergeant JA, Swaab DF. Pain processing in dementia and its relation to neuropathology. Lancet Neurol. 2003;2(11):677-686.

16. Willis WD, Westlund KN. Neuroanatomy of the pain system and the pathways that modulate pain. J Clin Neurophysiol. 1997;14:2-31.

17. Scherder E, Wolters E, Polman C, Sergeant J, Swaab D. Pain in Parkinson's disease and multiple sclerosis: its relation to the medial and lateral pain systems. Neurosci Biobehav Rev. 2005;29(7):1047-1056.

18. Fiest KM, Fisk JD, Patten SB, et al. Comorbidity is associated with pain-related activity limitations in multiple sclerosis. Mult Scler Relat Disord. 2015;4(5):470-476.

19. Horton M, Rudick RA, Hara-Cleaver C, Marrie RA. Validation of a self-report comorbidity questionnaire for multiple sclerosis. Neuroepidemiology. 2010;35(2):83-90.

20. Penner IK, Rausch M, Kappos L, Opwis K, Radu EW. Analysis of impairment related functional architecture in MS patients during performance of different attention tasks. J Neurol. 2003;250(4):461-472.

21. Scherder EJ, Bouma A. Visual analogue scales for pain assessment in Alzheimer's disease. Gerontology. 2000;46(1):47-53.

22. Benedetti F, Vighetti S, Ricco C, et al. Pain threshold and tolerance in Alzheimer's disease. Pain. 1999;80(1-2):377-382.

23. Folstein MF, Folstein SE, McHugh PR. Mini-mental state: a practical method for grading the cognitive state of patients for the clinician. J Psychiatr Res. 1975;12:189-198.

24. Lindeboom J, Jonker C. Amsterdamse Dementie Screening (ADS6). Lisse: Swets \& Zeitlinger; 1989.

25. Wilson B, Cockburn J, Baddeley A. The Rivermead Behavioural Memory Test. Titchfield: Thames Valley Test Company; 1987.

26. Wilson B, Alderman N, Burgess PW, Emslie H, Evans JJ. Behavioural Assessment of the Dysexecutive Syndrome. Bury St Edmunds, England: Thames Valley Test Company; 1996.

27. Scherder R, Kant N, Wolf E, Pijnenburg ACM, Scherder E. Pain and cognition in multiple sclerosis. Pain Med. 2017;18(10):1987-1998.

28. Gorczyca R, Filip R, Walczak E. Psychological aspects of pain. Ann Agric Environ Med. 2013;1:23-27.

29. Beck AT, Ward CH, Mendelson M, Mock J, Erbaugh J. An inventory for measuring depression. Arch Gen Psychiat. 1961;4:561-571.
30. Aikens JE, Reinecke MA, Pliskin NH, et al. Assessing depressive symptoms in multiple sclerosis: is it necessary to omit items from the original Beck Depression Inventory? J Behav Med. 1999;22(2):127-142.

31. Derogatis LR, Rickels K, Rock AF. The SCL-90 and the MMPI: a step in the validation of a new self-report scale. Brit J Psychiat. 1976;128: 280-289.

32. Arrindell WA, Ettema JHM. SCL-90, Handleiding bij een multidimensionele psychopathologie-indicator. Lisse: Swets \& Zeitlinger; 1986.

33. Kloot van der WA, Oostendorp RAB, Meij van der J, Heuvel van den J. De Nederlandse versie van McGill pain questionnaire: een betrouwbare pijnvragenlijst. Ned Tijdschr Geneeskd. 1995;139(13):669-673.

34. McGrath PA, Seifert CE, Speechly KN, Booth JC, Stitt L, Gibson MC. A new analogue scale for assessing children's pain: an initial validation study. Pain. 1996;64(3):435-443.

35. Kim EJ, Buschmann MT. Reliability and validity of the Faces Pain Scale with older adults. Int J Nurs Stud. 2006;43:447-456.

36. Perrot S, Javier RM, Marty M, Le Jeunne C, Laroche F; CEDR (Cercle d'Etude de la Douleur en Rhumatologie France), French Rheumatological Society, Pain Study Section. Is there any evidence to support the use of anti-depressants in painful rheumatological conditions? Systematic review of pharmacological and clinical studies. Rheumatology (Oxford). 2008;47(8):1117-1123.

37. Tetsunaga T, Tetsunaga T, Tanaka M, Ozaki T. Efficacy of tramadolacetaminophen tablets in low back pain patients with depression. J Orthop Sci. 2015;20(2):281-286.

38. Feinstein A, Freeman J, Lo AC. Treatment of progressive multiple sclerosis: what works, what does not, and what is needed. Lancet Neurol. 2015;14(2):194-207.

39. Marrie RA, Patten SB, Tremlett H, et al; CIHR Team in the Epidemiology and Impact of Comorbidity on Multiple Sclerosis. Sex differences in comorbidity at diagnosis of multiple sclerosis: a population-based study. Neurology. 2016;86(14):1279-1286.

40. Mitsonis CI, Potagas C, Zervas I, et al. The effects of stressful life events on the course of multiple sclerosis: a review. Int JNeurosci. 2009;119(3): 315-335.

41. Janssen SA, Spinhoven P, Arntz A. The effects of failing to control pain: an experimental investigation. Pain. 2004;107(3):227-233.

42. Kenner M, Menon U, Elliott DG. Multiple sclerosis as a painful disease. Int Rev Neurobiol. 2007;79:303-321.

43. Pöllmann W, Feneberg W. Current management of pain associated with multiple sclerosis. CNS Drugs. 2008;22(4):291-324.

44. Daniali SS, Shahnazi H, Kazemi S, Marzbani E. The effect of educational intervention on knowledge and self-efficacy for pain control in patients with multiple sclerosis. Mater Sociomed. 2016;28(4):283-287.

45. Oertel BG, Preibisch C, Wallenhorst T, et al. Differential opioid action on sensory and affective cerebral pain processing. Clin Pharmacol Ther. 2008;83(4):577-588.

46. Hemington KS, Cheng JC, Bosma RL, Rogachov A, Kim JA, Davis KD. Beyond negative pain-related psychological factors: resilience is related to lower pain affect in healthy adults. J Pain. 2017;18(9):1117-1128.

47. Carvalho IG, Bertolli ED, Paiva L, Rossi LA, Dantas RA, Pompeo DA. Anxiety, depression, resilience and self-esteem in individuals with cardiovascular diseases. Rev Lat Am Enfermagem. 2016;24:e2836.

48. Kurtzke, JE. Rating neurologic impairment in multiple sclerosis. An expanded disability status scale (EDSS). Neurology. 1983;33: 1444-1452.

49. Foley PL, Vesterinen HM, Laird BJ, et al. Prevalence and natural history of pain in adults with multiple sclerosis: systematic review and meta-analysis. Pain. 2013;154(5):632-642.

50. Zakzanis KK. Distinct neurocognitive profiles in multiple sclerosis subtypes. Arch Clin Neuropsychol. 2000;15(2):115-136.

51. Stenager E, Knudsen L, Jensen K. Acute and chronic pain syndromes in multiple sclerosis. A 5-year follow-up study. Ital J Neurol Sci. 1995;16(9):629-632.

52. Aupperle RL, Beatty WW, Shelton Fde N, Gontkovsky ST. Three screening batteries to detect cognitive impairment in multiple sclerosis. Mult Scler. 2002;8(5):382-389. 
53. Langdon DW, Amato MP, Boringa J, et al. Recommendations for a Brief International Cognitive Assessment for Multiple Sclerosis (BICAMS). Mult Scler. 2012;18(6):891-898.

54. Ayache SS, Chalah MA. Fatigue in multiple sclerosis - insights into evaluation and management. Neurophysiol Clin. 2017;47(2): $139-171$.
55. Kratz AL, Murphy SL, Braley TJ. Pain, fatigue, and cognitive symptoms are temporally associated within- but not across-days in multiple sclerosis. Arch Phys Med Rehabil. 2017;98(11):2151-2159.

56. O'Connor AB, Schwid SR, Herrmann DN, Markman JD, Dworkin RH. Pain associated with multiple sclerosis: systematic review and proposed classification. Pain. 2008;137(1):96-111.

\section{Publish your work in this journal}

The Journal of Pain Research is an international, peer reviewed, open access, online journal that welcomes laboratory and clinical findings in the fields of pain research and the prevention and management of pain. Original research, reviews, symposium reports, hypothesis formation and commentaries are all considered for publication.
Dovepress

The manuscript management system is completely online and includes a very quick and fair peer-review system, which is all easy to use. Visit http://www.dovepress.com/testimonials.php to read real quotes from published authors. 